\title{
Special issue on magnetic resonance imaging biomarkers of renal disease
}

\author{
Paul Hockings ${ }^{1} \cdot$ Christoffer Laustsen ${ }^{2} \cdot$ Jaap A. Joles ${ }^{3} \cdot$ Patrick B. Mark $^{4} \cdot$ Steven Sourbron $^{5}$
}

Published online: 3 January 2020

(c) European Society for Magnetic Resonance in Medicine and Biology (ESMRMB) 2020

The idea of using MRI to assess renal function dates back to the early 1980s, when Runge et al. demonstrated that serial MRI after contrast agent injection revealed temporal patterns that were able to diagnose acute changes in renal function [1]. In the same period, Pettigrew et al. demonstrated that fast imaging sequences could isolate renal perfusion [2], and concluded that "in the future, MR imaging [...] may simultaneously provide good morphological detail and the type of physiologic information currently offered noninvasively by nuclear medicine techniques". Ever since, functional renal MRI has been an active field of research within the MRI community. Commonly cited methods include diffusionweighted imaging [3], diffusion-tensor imaging [4], bloodoxygenation-level-dependent MRI [5] and arterial spin labelling [6], but many others have shown a relevant signal in the kidney: phase-contrast, T1-mapping, T2-mapping, sodium, magnetisation transfer, chemical exchange saturation transfer, spectroscopy, rotating frame relaxation, elastography, volumetry, quantitative susceptibility mapping and hyperpolarised MRI.

For 30 years, these ideas were largely confined to the MR physics and radiology community, but the past few years have seen a rapid increasing interest by nephrologists. To a large extent, this is driven by the ever growing burden of chronic kidney disease (CKD) and the well-recognised need

Steven Sourbron

s.sourbron@sheffield.ac.uk

1 Antaros Medical, Mölndal, Sweden

2 Department of Clinical Medicine, The MR Research Centre, Aarhus University, Aarhus, Denmark

3 Department of Nephrology and Hypertension, University Medical Centre Utrecht, Utrecht, The Netherlands

4 Institute of Cardiovascular and Medical Sciences, University of Glasgow, Glasgow, UK

5 Department of Imaging, Infection, Immunity and Cardiovascular Disease, University of Sheffield, 18 Claremont Crescent, Sheffield S10 2TA, UK for "novel prognostic biomarkers that help to predict future risk and understand the underlying molecular mechanisms" [7]. One possible avenue lies in the identification of novel biomarkers in blood or urine, but these have so far failed to produce a clear solution [8]. It is increasingly recognised that MRI biomarkers may be part of the solution due to their ability to probe different pathophysiological hallmarks of CKD progression [9]. Despite known limitations in terms of biological specificity, it appears plausible that a direct observation of parenchymal changes in situ can pick up disease progression well before it manifests itself downstream in blood or urine.

These converging interests of MR physicists, radiologists, nephrologists, drug developers, transplant surgeons, physiologists and pathologists have given rise to a dynamic and multi-disciplinary community of researchers with a common interest in renal MRI biomarkers. In Europe, an international research network was founded in 2017 (www.renalmri.org), and only recently, in October 2019, a third international meeting on renal MRI was held attracting over 200 scientists from across these disciplines [10]. As a result of this broadening interest, MRI biomarkers are also increasingly visible in the nephrology literature [11], with one recent study hinting at a potential role in the long-standing problem of predicting disease progression [12].

This special issue on MRI biomarkers of renal disease is intended to offer a cross section of the ongoing developments in this area, and to encourage a more coordinated approach to MRI biomarker development in order to generate the evidence levels required by regulators [13].

The four review papers in this issue cover ongoing areas of research including relatively well-established methods (Caroli et al.) and entirely novel contrast mechanisms (Laustsen et al.), image processing (Zoellner et al.) and clinical applications (Schutter et al.). The original work in this issue is representative of the wider field and includes the application of MRI to improve patient management (Serai et al.) and our understanding of disease progression (Van 
Raalte et al.), studies on sequence optimisation (Harteveldt et al., Miyazaki et al.) and image processing methods (Rankin et al., Li et al.) that may inform future technical recommendations and technical developments that can open up new avenues of translational research (Boehmert et al.).

This special issue places a particular emphasis on research coordination, and presents a rigorous process developed by the community to generate expert consensus on technical aspects (Mendichovszky et al.). Four papers present the results of applying this process to the most common contrast mechanisms and have jointly generated over 160 consensus statements (Nery et al., Dekkers et al., Ljimani et al., Bane et al.). Apart from identifying areas where consensus already exists, this process has also served to identify research priorities by highlighting areas where experts are currently unable to agree on a recommendation. Our hope is that this mechanism will ultimately cause an alignment of the methods for measuring renal MRI biomarkers and create the necessary conditions for international harmonisation. This in turn will allow clinical trials to be scaled up to the levels needed to demonstrate the utility of this promising new paradigm in the diagnosis of renal disease.

Funding Steven Sourbron is funded by Innovative Medicines Initiative (115974) and Medical Research Council (MR/R02264X/1).

\section{Compliance with ethical standards}

Conflict of interest Paul Hockings is director of Imaging at Antaros Medical. Other authors declare no conflict of interest.

Ethical approval This paper does not report studies with animals or human participants.

\section{References}

1. Runge VM, Clanton JA, Herzer WA, Gibbs SJ, Price AC, Partain CL et al (1984) Intravascular contrast agents suitable for magnetic resonance imaging. Radiology 153:171-173
2. Pettigrew RI, Avruch L, Dannels W, Coumans J, Bernardino ME (1986) Fast-field-echo MR imaging with Gd-DTPA: physiologic evaluation of the kidney and liver. Radiology 160:561-563

3. Müller MF, Prasad PV, Bimmler D, Kaiser A, Edelman RR (1994) Functional imaging of the kidney by means of measurement of the apparent diffusion coefficient. Radiology 193:711-715

4. Ries M, Jones RA, Basseau F, Moonen CTW, Grenier N (2001) Diffusion tensor MRI of the human kidney. J Magn Reson Imaging 14:42-49

5. Prasad PV, Edelman RR, Epstein FH (1996) Noninvasive evaluation of intrarenal oxygenation with BOLD MRI. Circulation 94:3271-3275

6. Karger N, Biederer J, Lûsse S, Grimm J, Steffens JC, Heller M et al (2000) Quantitation of renal perfusion using arterial spin labeling with FAIR-ULFARE. Magn Reson Imaging 18:641-647

7. Levin A, Tonelli M, Bonventre J, Coresh J, Donner JA, Fogo AB et al (2017) Global kidney health 2017 and beyond: a roadmap for closing gaps in care, research, and policy. Lancet 390:1888-1917

8. Heinzel A, Kammer M, Mayer G, Reindl-Schwaighofer R, Hu K, Perco P et al (2018) Validation of plasma biomarker candidates for the prediction of eGFR decline in patients with type 2 diabetes. Diabetes Care 41:1947-1654

9. Selby NM, Blankestijn PJ, Boor P, Combe C, Eckardt K-U, Eikefjord E et al (2018) Magnetic resonance imaging biomarkers for chronic kidney disease: a position paper from the European Cooperation in Science and Technology Action PARENCHIMA. Nephrol Dial Transplant. 33:i4-i14

10. Francis S, Selby N, Taal M (2019) 3d international meeting on renal MRI [Internet]. https://www.nottingham.ac.uk/research/ groups/spmic/research/uk-renal-imaging-network/3rd-renal -symposium/3rd-international-symposium-on-functional-renal -imaging.aspx. Accessed 26 Dec 2019

11. Caroli A, Pruijm M, Burnier M, Selby NM (2018) Functional magnetic resonance imaging of the kidneys: where do we stand? The perspective of the European COST action PARENCHIMA. Nephrol Dial Transplant 33:ii1

12. Sugiyama K, Inoue T, Kozawa E, Ishikawa M, Shimada A, Kobayashi $\mathrm{N}$ et al (2018) Reduced oxygenation but not fibrosis defined by functional magnetic resonance imaging predicts the long-term progression of chronic kidney disease. Nephrol Dial Transplant. https://doi.org/10.1093/ndt/gfy324

13. Thompson A (2015) Clinical review of PKD outcomes consortium biomarker qualification submission [Internet]. https://www.fda. gov/media/93159/download. Accessed 26 Dec 2019

Publisher's Note Springer Nature remains neutral with regard to jurisdictional claims in published maps and institutional affiliations. 